\title{
Disentanglement of pure bipartite quantum states by local cloning
}

\author{
Somshubhro Bandyopadhyay ${ }^{a}$ 円, Guruprasad $\operatorname{Kar}^{b}$ and Anirban Roy $^{b}$ \\ ${ }^{a}$ Department of Physics, Bose Institute, 93/1 A.P.C. Road, Calcutta -700009, \\ India \\ ${ }^{b}$ Physics and Applied Mathematics Unit, Indian Statistical Institute, 203 B.T. \\ Road, Calcutta -700035, India
}

\section{Corresponding author}

Somshubhro Bandyopadhyay

Department of Physics

Bose Institute

93/1 A.P.C. Road

Calcutta - 700009

INDIA

email: dhom@boseinst.ernet.in

Telephone: 91 - 33 - 350 - 2402/03, 91 - 33 - 350-6702

FAX: $91-33-350-6790$

\begin{abstract}
We discuss disentanglement of pure bipartite quantum states within the framework of the schemes developed for entanglement splitting and broadcasting of entanglement.
\end{abstract}

\footnotetext{
1 email: dhom@boseinst.ernet.in
} 


\section{Introduction}

A composite quantum system consisting of two subsystems is said to be entangled or inseparable if in general the density matrix cannot be written as

$$
\rho=\sum w_{i} \rho_{i}^{(1)} \otimes \rho_{i}^{(2)}
$$

where the positive weights $w_{i}$ satisfy $\sum w_{i}=1$ and $\rho^{(1)}=\operatorname{Tr}_{2}(\rho)$ and $\rho^{(2)}=$ $\operatorname{Tr}_{1}(\rho)$ are the reduced density matrices of the subsystems. Therefore the density matrix of a separable or disentangled system can always be written in the form (1). So the question is whether there exisits any universal transformation acting on any given arbitrary entangled state $\rho$ transforms it into a state that can be written in the form (1). If really such transformation exists then we have an ideal disentanglement machine $(D M)$. Thus disentanglement is the process which transforms an inseparable or entangled state (consisting of two qubits) into a separable one such that the reduced density operators of the individual subsystems remain unchanged.

First we give two definitions of disentanglement [1,2]:

Definition 1 - Disentanglement is the process that transforms a state of two (or more) subsystems into an unentangled state (in general, a mixture of product states) such that the reduced density matrices of each of the subsystems are unaffaected.

Definition 2 - Disentanglement into a tensor product state is the process that transforms a state of two (or more) subsystems into a tensor product of the two reduced density matrices.

Note that the second definition is a special case of the first one.

Recently Terno [2] showed that universal disentanglement into product states is impossible. Tal Mor [1] investigated the possibility of universal disentanglement into separable states where he showed that a universal disentangling machine cannot exist. We now briefly describe what the above stated two results actually mean.

The first result (Terno[2]) tells us that

$$
\rho^{e n t} \underline{D M} \triangleright \rho^{(1)} \otimes \rho^{(2)}
$$

is not allowed.

The second result (T. Mor [1]) negates the possibilty of

$$
\rho^{\text {ent }} \underline{D M} \triangleright \rho^{\text {disent }}
$$

such that

$$
\rho^{(i)}=\operatorname{Tr}_{j}\left(\rho^{\text {ent }}\right)=\operatorname{Tr}_{j}\left(\rho^{\text {disent }}\right) ; \quad i \neq j ; \quad i, j=1,2
$$

So the question is then how well we can disentangle a pure bipartite quantum state? The answer lies in the possibilty of constructing an $U D M$ such that the reduced density operators of $\rho^{\text {disent }}$ are close to the reduced density operators 
corresponding to $\rho^{\text {ent }}$. What is partcularly meant by closeness will be clear from the following discussion. We require that the reduced state operators of the subsystems after disentanglement should remain in a scaled form (isotropy requirement) i.e. $\rho_{a d}^{(i)}=\eta \rho_{b d}^{(i)}+\left(\frac{1-\eta}{2}\right) I$ f, then $\eta$ being the scaling parameter (can take values between 0 and 1 in principle) stands as a measure of closeness. For example $\eta=1$ tells us that the reduced state operator after disentanglement hasn't changed whereas $\eta=0$ gives the information that the subsystems have become totally random after disentanglement etc.

Keeping in mind the above constraints we discuss how the schemes developed for entanglement splitting [3] and broadcasting of entanglement [4, 5] can be used for disentangling a pure entangled state.

\section{Disentanglement of a two qubit pure entangled state}

In what follows we discuss how disentanglement of a pure two qubit entangled state can be achieved by local cloning of the qubits within the existing schemes of entanglement splitting and broadcasting of entanglement. Before that we recall what precisely we want to achieve. Our aim is to have

$$
\rho^{\text {ent }} \underline{D M} \triangleright \rho^{\text {disent }}
$$

such that $\quad \rho_{b d}^{(i)}=\operatorname{Tr}_{j}\left(\rho^{\text {ent }}\right)$ is close to $\rho_{a d}^{(i)}=\operatorname{Tr}_{j}\left(\rho^{\text {disent }}\right) ; \quad i \neq j ; \quad i, j=1,2$.

In particular we want

$$
\rho_{a d}^{(i)}=\eta \rho_{b d}^{(i)}+\left(\frac{1-\eta}{2}\right) I
$$

such that $\eta$ takes the maximum possible value.

\section{1. Disentanglement by local cloning of one qubit}

First we consider how we can disentangle a two qubit pure entangled state by local cloning of any one qubit. The local cloning of a single qubit of a two particle entangled state forms the basis of entanglement splitting [3] that can be defined as the process by which any one of a two party entangled system transfers a part of his entanglement to a third party. Suppose we have two parties $x$ and $y$ sharing an entangled state of two qubits given by

$$
|\psi\rangle=\alpha|00\rangle_{x y}+\beta|11\rangle_{x y}
$$

The first qubit belongs to $x$ and the second belongs to $y$ as usual. Now the qubit belonging to any one ( say $x$ ) of the two is cloned and let the two copies be $x_{1}$ and $x_{2}$. This gives rise to a composite system $\rho_{x_{1} x_{2} y}$ consisting of three qubits. Tracing out one of the copies $x_{i}(i=1,2)$ produces a two qubit composite system which is inseparable under certain conditions. This is the basic concept of entanglement splitting. Note that in this process nothing is done to the qubit

\footnotetext{
${ }^{2} a d$ and $b d$ stands for after disentanglement and before disentanglement respectively
} 
that belongs to $y$. Thus the reduced density matrix corresponding to $y$ remains unchanged.

Consider the following universal cloning transformation for local copying of the subsystem $x$, defined by

$$
\begin{aligned}
& U|0\rangle|\rangle|Q\rangle=a|00\rangle|A\rangle+b(|01\rangle+|10\rangle)|B\rangle \\
& U|1\rangle|\rangle|Q\rangle=a|11\rangle \widetilde{|A\rangle}+b(|01\rangle+|10\rangle) \widetilde{|B\rangle}
\end{aligned}
$$

where |\rangle denotes the blank qubit supplied to the cloner, $|Q\rangle$ denotes the initial state of the quantum copier (ancilla), $|A\rangle,|B\rangle,|\widetilde{A}\rangle, \widetilde{|B\rangle}$ are the normalized ancilla output states. The coefficients $a$ and $b$ are in general complex. The following conditions hold from unitarity, isotropy and symmetry requirements for an universal quantum cloner [6]

$$
\begin{gathered}
|a|^{2}+2|b|^{2}=1 \\
\langle B| \widetilde{B\rangle}=\langle A \mid B\rangle=\widetilde{\langle A \mid B\rangle}=0
\end{gathered}
$$

The fidelity of the above universal quantum cloner defined by the transformations (4) and (5) along with the conditions (6) and (7), is given by

$$
F=\frac{1}{2}(1+\eta)
$$

where the reduction factor (also known as the Black Cow factor [7]) $\eta$ is given by $[6]$

$$
\left.\left.\eta=|a|^{2}=\operatorname{Re}\left(a b^{*} \widetilde{\langle B|} A\right\rangle+a^{*} b \widetilde{\langle A|} B\right\rangle\right)
$$

Choosing, $\widetilde{\langle B}|A\rangle=\widetilde{\langle A}|B\rangle=1$ one obtains the optimal quantum cloner [8,9] for which $\eta=2 / 3$. Thus a less optimal quantum cloner but nevertheless universal (isotropic) can be constructed by varying the scalar product of the ancilla output states.

We now apply this cloning transformation to copy the subsystem $x$ to produce the copies $x_{1}$ and $x_{2}$. After tracing out the cloning machine part and any one of the copies the resulting density matrix is given by

$$
\begin{gathered}
\rho_{x_{i} y}=\left(\frac{1+\eta}{2}\right)\left(\alpha^{2}|00\rangle\left\langle 00\left|+\beta^{2}\right| 11\right\rangle\langle 11|\right)+\alpha \beta \eta(|00\rangle\langle 11|+| 11\rangle\langle 00|) \\
+\left(\frac{1-\eta}{2}\right)\left(\alpha^{2}|01\rangle\left\langle 01\left|+\beta^{2}\right| 10\right\rangle\langle 10|\right) \quad i=1,2
\end{gathered}
$$

Applying the Peres - Horodecki theorem $[10,11]$ to test the inseparability of $\rho_{x_{i} y}$ it turns out that the state is inseparable for all values of $\alpha$ provided $\eta>1 / 3$ (Note that upper bound of $\eta$ is $2 / 3$ ). Thus it is possible to achieve disentanglement of any arbitrary pure two particle entangled state provided we employ an universal (isotropic) cloner whose fidelity $F \leq 2 / 3$ (recall that $\eta$ and $F$ are related by Eq. 8) to copy one of the qubits. Now that our requirement is also to have reduced density matrices of the disentangled state as close as possible 
to those of the entangled one, we note that the reduced density matrix of the subsystem $y$ is unaltered whereas that of the subsystem $x$ is changed. It is clear that the subsystem $x$ is copied rather poorly since the maximum fidelity with which it has to be copied to achieve disentanglement is $2 / 3$. Thus after disentanglement by this process, although one suceeds in keeping one subsystem unchanged but ends up with a rather poor copy of the other. Let us summarise the results of the preceeding section.

1. It is possible to disentangle any arbitrary bipartite entangled state by applying local cloning on one of its qubits provided the reduction factor of the isotropic cloner is less than or equal to $1 / 3$ (i.e. fidelity $F \leq 2 / 3$ ).

2. After disentanglement the reduced density matices of the subsystems are given by

$$
\begin{gathered}
\rho_{a d}^{(y)}=\rho_{b d}^{(y)} \\
\rho_{a d}^{(x)}=\eta \rho_{b d}^{(x)}+\left(\frac{1-\eta}{2}\right) I \quad \text { where } \eta_{\max }=1 / 3 .
\end{gathered}
$$

\section{2. Disentanglement by local cloning of both the qubits}

In the previous section we showed how to disentangle pure states by applying local cloning operation on one of the qubits. In this section we use the concept of broadcasting quantum inseparability via local copying, first shown to be possible by Buzek et al [4]. First we briefly sketch the essentials of broadcasting of entanglement where the entanglement originally shared by a single pair is transferred into two less entangled pairs using only local operations. Suppose two distant parties $a_{1}$ and $a_{2}$ share an entangled two qubit state

$$
|\psi\rangle=\alpha|00\rangle_{a_{1} a_{2}}+\beta|11\rangle_{a_{1} a_{2}}
$$

where $\alpha^{2}+\beta^{2}=1$ and $\alpha, \beta$ are real.

The first qubit belongs to $a_{1}$ and the second belongs to $a_{2}$. Each of the two parties now performs local cloning operations on their own qubit. It turns out that for some values of $\alpha$,

(a) non local output states are inseparable, and

(b) local output states are separable

hold simultaneously. Buzek et al. [4] used optimal quantum cloners for local copying of the subsystems and showed that the nonlocal outputs are inseparable if

$$
\frac{1}{2}-\frac{\sqrt{39}}{16} \leq \alpha^{2} \leq \frac{1}{2}+\frac{\sqrt{39}}{16}
$$

Now consider the same system defined by (10). The two subsystems $a_{i}(i=$ $1,2)$ are locally copied according to the cloning transformations (4) and (5) to produce output two systems $b_{i}(i=1,2)$. The local output state of a copier is given by the density operator 


$$
\widehat{\rho}_{a_{i} b_{i}}^{(\text {out })}=\alpha^{2} \eta|00\rangle\left\langle 00\left|+\beta^{2} \eta\right| \mid 11\right\rangle\langle 11|+(1-\eta)|+\rangle\langle+\}^{3}
$$

and the nonlocal output is described by the density operator

$$
\begin{aligned}
\widehat{\rho}_{a_{i} b_{j}}^{(\text {out })}= & {\left[\alpha^{2} \eta+\left(\frac{1-\eta}{2}\right)^{2}\right]|00\rangle\left\langle 00\left|+\left[\beta^{2} \eta+\left(\frac{1-\eta}{2}\right)^{2}\right]\right| 11\right\rangle\langle 11| } \\
+\left(\frac{1-\eta^{2}}{4}\right)(|01\rangle\langle 01|+| 10\rangle\langle 10|)+\alpha \beta \eta^{2}(|00\rangle\langle 11|+| 11\rangle\langle 00|) & \quad i \neq j ; \quad i, j=1,2
\end{aligned}
$$

It follows from the Peres-Horodecki theorem $[10,11]$ that $\widehat{\rho}_{a_{i} b_{j}}^{(o u t)}$ is inseparable if

$$
\frac{1}{2}-\left[\frac{1}{4}-\frac{\left(1-\eta^{2}\right)^{2}}{16 \eta^{4}}\right]^{1 / 2} \leq \alpha^{2} \leq \frac{1}{2}+\left[\frac{1}{4}-\frac{\left(1-\eta^{2}\right)^{2}}{16 \eta^{4}}\right]^{1 / 2}
$$

The requirement that $\left[\frac{1}{4}-\frac{\xi^{2}(1-\xi)^{2}}{(1-2 \xi)^{4}}\right]^{1 / 2}$ has to be positive otherwise the domain of $\alpha^{2}$ would be meaningless leads to the lower bound of $\eta$,

$$
\eta \geq \sqrt{\frac{1}{3}}
$$

The upper bound is of course $2 / 3$ corresponding to the optimal quantum cloner.

Again applying the Peres-Horodecki theorem it is easy to obtain that $\widehat{\rho}_{a_{i} b_{i}}^{(\text {out })}$ is separable if

$$
\frac{1}{2}-\left\{\frac{1}{4}-\frac{(1-\eta)^{2}}{4 \eta^{2}}\right\}^{1 / 2} \leq \alpha^{2} \leq \frac{1}{2}+\left\{\frac{1}{4}-\frac{(1-\eta)^{2}}{4 \eta^{2}}\right\}^{1 / 2}
$$

As one can observe comparing (14) and (16) that $\widehat{\rho}_{a_{i} b_{i}}^{(\text {out })}$ is separable if $\widehat{\rho}_{a_{i} b_{j}}^{(o u t)}$ is inseparable.

Here our interest lies in the possibilty of disentangling the state (10) and therefore we note from inequality (15) that the state (13) becomes separable for all $\alpha$ when $\eta<1 / \sqrt{3}$. But when $\frac{1}{\sqrt{3}} \leq \eta \leq \frac{2}{3}$ its still possible to disentangle the state (10) but not for all $\alpha$ as is clear from (14). Besides our objective is also to copy the subsystem in the best possible way for which one has to use optimal quantum cloners for local copying. In that case if and only if $\alpha$ lies outside the range given by (11) the final state becomes disentangled with the best possible reduced density matrices of the subsystems. Now let us summarise the main results ,

1. An arbitrary bipartite pure entangled state can be disentangled by local cloning of the individual subsystems provided the reduction factor of the isotropic cloners used is less than $1 / \sqrt{3}$ (ie. fidelity $F<\frac{\sqrt{3}+1}{2 \sqrt{3}}$ ).

2. After disentanglement of the original state the reduced density matrices of the subsystems are given by

$$
\rho_{a d}^{\left(a_{i}\right)}=\eta \rho_{b d}^{\left(a_{i}\right)}+\left(\frac{1-\eta}{2}\right) I \quad i=1,2
$$

\footnotetext{
${ }^{3}|+\rangle=\frac{1}{\sqrt{2}}(|01\rangle+|10\rangle)$
} 
where $\eta<1 / \sqrt{3}$.

Its clear from the above discussions that its impossible to disentangle an arbitrary pure two particle entangled state by using optimal quantum cloners to copy the subsystems locally such that qualitatively best possible reduced density operators of the subsystems can be obtained.

Here we observe that all the above discussions particularly hold good when we use a isotropic quantum cloner of $1 \rightarrow 2$ type. So we can as well look for the possibility of disentanglement using an optimal cloner of type $1 \rightarrow M$ for some $M(M>2)$ to copy the qubits locally. Since we know that quality of copies decrease with increasing $M$, therefore we should look for the smallest value of $M(M>2)$ such that applying an optimal $1 \rightarrow M$ quantum cloner to copy the qubits locally we will be able to disentangle a pure state. It has already been shown [5] that producing three copies of any pure entanglement by applying $1 \rightarrow 3$ optimal quantum cloner for local copying of the individual subsystems is forbidden. So the two criteria are satisfied for $M=3$. The important thing to note in this case is the fidelity of an optimal $1 \rightarrow 3$ quantum cloner which is $7 / 9$. We have seen earlier that disentanglement of any pure state can be achieved if we clone the qubits locally by an $1 \rightarrow 2$ isotropic cloner of fidelity $F<\frac{\sqrt{3}+1}{2 \sqrt{3}}$ (see Eq. (8) and the discussion after inequality (16)) whereas the fidelity of each local qubit in the later case being $7 / 9$. Although disentanglement is achieved in both the cases for any arbitrary pure entangled state the previous one is better since $\frac{\sqrt{3}+1}{2 \sqrt{3}}>7 / 9$ implying that locally individual systems are better copied.

Till now we have described how one is able to disentangle a pure two qubit entangled state working within the existing schemes of entanglement splitting and broadcasting of entanglement. One distinct advantage of the first scheme (where we have made use of the concept of entanglement splitting) is that the state of one of the subsystems remains unchanged though the copy of the other subsystem is rather poor $\left[\eta_{\text {threshold }}=1 / 3 ;\right.$ Fidelity threshold $=$ $\left.\frac{1}{2}(1+\eta)=2 / 3=0.666\right]$ as compared to the second where both the subsystems undergo change in their respective states but the copies being better $\left(\eta_{\text {threshold }}<1 / \sqrt{3} ;\right.$ Fidelitythreshold $\left.<\frac{1}{2}(1+\eta)=\frac{\sqrt{3}+1}{2 \sqrt{3}}=0.788\right)$. Nevertheless the second scheme allows the possibilty of using the optimal cloner provided the parameter $\alpha$ of the original entangled state lies outside the range specified by (11). So which scheme is better can probably be best justified from the motivation of a given problem where disentanglement is required.

\section{Conclusion}

Since universal disentanglement into separable states is not allowed we have explored how well one can approximate universal disentanglement of a pure 
bipartite quantum state. In other words we have tried to answer possibly "how good" an universal disentanglement machine can be since construction of a perfect universal disentanglement machine is forbidden. We have shown that working with less optimal but isotropic quantum cloner within the framework of entanglement splitting and broadcasting of entanglement it is possible to disentangle a pure bipartirte quantum state.

\section{References}

[1] T. Mor, quant-ph/9812020.

[2] D. Terno, quant-ph/9811036.

[3] D. Bruß, quant-ph/9902023.

[4] V. Buzek, V. Vedral, M. B. Plenio, P. L. Knight and M. Hillery, Phys. Rev. A 55 (1997) 3327.

[5] S. Bandyopadhyay and G. Kar, quant-ph/9902073.

[6] D. Bruß, D. P. DiVincenzo, A. Ekert, C. A. Fuchs, C. Macchiavello and J. A. Smolin, Phys. Rev. A 57 (1998) 2368.

[7] R. Werner, quant-ph/9804001.

[8] V. Buzek and M. Hillery, Phys. Rev. A 54 (1996) 1844.

[9] N. Gisin and S. Massar, Phys. Rev. Lett. 79 (1997) 2153.

[10] A. Peres, Phys. Rev. Lett. 77 (1996) 1413.

[11] M. Horodecki, P. Horodecki and R. Horodecki, Phys. Lett. A 223 (1996) 1. 